\title{
Distances from Bloch functions to some Möbius invariant function spaces in the unit ball of $\mathbb{C}^{n}$
}

\author{
Wen Xu \\ (Communicated by Miroslav Englǐ̌)
}

2000 Mathematics Subject Classification. 32A18, 32A37.

Keywords and phrases. Bloch space, distance, $Q_{s}$ space, Besov space.

\begin{abstract}
Distance formulae from Bloch functions to some Möbius invariant function spaces in the unit ball of $\mathbb{C}^{n}$ such as $Q_{s}$ spaces, little Bloch space $\mathcal{B}_{0}$ and Besov spaces $B_{p}$ are given.
\end{abstract}

\section{Introduction}

Let $B$ be the unit ball of $\mathbb{C}^{n}$ with boundary $S$, let $d \nu$ denote the Lebesgue measure on $B$ such that $\nu(B)=1$ and let $d \sigma$ be the rotation invariant positive normalized measure on $S$, i.e. $\sigma(S)=1$. Let $d \lambda(z)=$ $\left(1-|z|^{2}\right)^{-(n+1)} d \nu(z)$. Then $d \lambda$ is Möbius invariant. For $\alpha>-1$, the weighted Lebesgue measure $d \nu_{\alpha}$ is defined by

$$
d \nu_{\alpha}(z)=c_{\alpha}\left(1-|z|^{2}\right)^{\alpha} d \nu(z)
$$

where

$$
c_{\alpha}=\frac{\Gamma(n+\alpha+1)}{n ! \Gamma(\alpha+1)}
$$


is a normalizing constant such that $d \nu_{\alpha}$ is a probability measure on $B$, i.e. $\nu_{\alpha}(B)=1$.

Let $H(B)$ be the class of all holomorphic functions in the unit ball $B$, and let $\operatorname{Aut}(B)$ be the group of biholomorphic automorphisms of $B$. For $a \in B$, let $\varphi_{a} \in \operatorname{Aut}(B)$ denote the Möbius transformation of $B$ which satisfies $\varphi_{a}(0)=a, \varphi_{a}(a)=0$ and $\varphi_{a} \circ \varphi_{a}=I$. Further, $1-\left|\varphi_{a}(z)\right|^{2}=\frac{\left(1-|a|^{2}\right)\left(1-|z|^{2}\right)}{|1-\langle z, a\rangle|^{2}}$ for all $z \in B$, where $\langle z, a\rangle$ is the usual inner product on $\mathbb{C}^{n}$.

For $f \in H(B), \nabla f=\left(\frac{\partial f}{\partial z_{1}}, \cdots, \frac{\partial f}{\partial z_{n}}\right)$ is called its complex gradient, $\mathcal{R} f(z)=\sum_{j} z_{j} \frac{\partial f}{\partial z_{j}}(z)$ is called its radial derivative, and $\widetilde{\nabla} f(z)=\nabla(f \circ$ $\left.\varphi_{z}\right)(0)$ is called its Möbius invariant gradient. It is known that (1$\left.|z|^{2}\right)|R f(z)| \leq\left(1-|z|^{2}\right)|\nabla f(z)| \leq|\widetilde{\nabla} f(z)|$ (cf. [8, Lemma 2.14]).

The invariant Green's function $G(z, a)$ of the unit ball $B$ is defined by $G(z, a)=g\left(\varphi_{a}(z)\right)$ (cf. [5]), where

$$
g(z)=\frac{n+1}{2 n} \int_{|z|}^{1}\left(1-t^{2}\right)^{n-1} t^{-2 n+1} d t
$$

In [3], the holomorphic function spaces $Q_{s}$ associated with the Green's function are introduced and studied. For $s>0, Q_{s}$ is defined by

$$
Q_{s}=\left\{f \in H(B): \sup _{a \in B} \int_{B}|\widetilde{\nabla} f(z)|^{2} G(z, a)^{s} d \lambda(z)<\infty\right\},
$$

and its subspace $Q_{s, 0}$ is defined by

$$
Q_{s, 0}=\left\{f \in H(B): \lim _{|a| \rightarrow 1} \int_{B}|\widetilde{\nabla} f(z)|^{2} G(z, a)^{s} d \lambda(z)=0\right\} .
$$

The Bloch space on $B$, denoted by $\mathcal{B}$, is the class of all functions $f \in H(B)$, which satisfy

$$
\|f\|_{1}=\sup _{z \in B}\left(1-|z|^{2}\right)|\mathcal{R} f(z)|<\infty .
$$

The little Bloch space $\mathcal{B}_{0}$ on $B$ is a subspace of $\mathcal{B}$, and $f \in \mathcal{B}_{0}$ if and only if

$$
\lim _{|z| \rightarrow 1}\left(1-|z|^{2}\right)|\mathcal{R} f(z)|=0
$$

Let

$$
\|f\|_{2}=\sup _{z \in B}\left(1-|z|^{2}\right)|\nabla f(z)|, \quad\|f\|_{3}=\sup _{z \in B}|\widetilde{\nabla} f(z)| .
$$


It is known that the above three semi-norms are equivalent (cf. [8]). Define

$$
\|f\|_{\mathcal{B}}=|f(0)|+\|f\|_{1}=|f(0)|+\sup _{z \in B}\left(1-|z|^{2}\right)|\mathcal{R} f(z)| .
$$

Then $\mathcal{B}$ is a Banach space with the norm $\|\cdot\|_{\mathcal{B}}$.

For $\alpha>-1$ and $p>0$, the weighted Bergman space $A_{\alpha}^{p}$ consists of holomorphic functions $f$ satisfying

$$
\|f\|_{p, \alpha}=\left(\int_{B}|f(z)|^{p} d \nu_{\alpha}(z)\right)^{\frac{1}{p}}<\infty .
$$

For a multi-index $m=\left(m_{1}, m_{2}, \cdots, m_{n}\right)$ with non-negative integers, we will employ the notation

$$
\frac{\partial^{m} f}{\partial z^{m}}=\frac{\partial^{|m|} f}{\partial z_{1}^{m_{1}} \cdots \partial z_{n}^{m_{n}}}
$$

where $|m|=m_{1}+m_{2}+\cdots+m_{n}$. For $0<p<\infty$, the Besov space $B_{p}$ (see $[8]$ ) is the class of holomorphic functions $f$ in $B$ such that the functions

$$
\left(1-|z|^{2}\right)^{N} \frac{\partial^{m} f}{\partial z^{m}}(z), \quad|m|=N
$$

all belong to $L^{p}(B, d \lambda)$, where $\mathrm{N}$ is any integer satisfying $p N>n$.

For two real parameters $\alpha$ and $t$ with the property that neither $n+\alpha$ nor $n+\alpha+t$ is a negative integer, if $f(z)=\sum_{k=0}^{\infty} f_{k}(z)$ is the homogeneous expansion of $f$, an invertible operator $\mathcal{R}^{\alpha, t}: H(B) \rightarrow H(B)$ is defined in [8] by

$$
\mathcal{R}^{\alpha, t} f(z)=\sum_{k=0}^{\infty} \frac{\Gamma(n+1+\alpha) \Gamma(n+1+\alpha+k+t)}{\Gamma(n+1+\alpha+t) \Gamma(n+1+\alpha+k)} f_{k}(z) .
$$

For $\zeta \in S$ and $\delta>0$, let $B(\zeta, \delta)=\{z \in B:|1-\langle z, \zeta\rangle|<\delta\}$. For a positive Borel measure $\mu$ on $B$, if

$$
\sup \left\{\frac{\mu(B(\zeta, \delta))}{\delta^{n p}}: \zeta \in S, \delta>0\right\}<\infty
$$

we call $\mu$ a $p$-Carleson measure; if

$$
\lim _{\delta \rightarrow 0} \frac{\mu(B(\zeta, \delta))}{\delta^{n p}}=0
$$

for $\zeta \in S$ uniformly, we call $\mu$ a vanishing $p$-Carleson measure. 
The purpose of this paper is to drive distance formulae from Bloch functions to some Möbius invariant function spaces on the unit ball which generalize the results of [7]. R. Zhao, in [7], takes advantage of the second inequality in Lemma 2.5 [2] while we use the third inequality. In fact, the second inequality is not feasible in our multidimensional case.

Throughout this paper, $\mathrm{C}$ denotes a positive constant and not necessarily the same at each occurrence.

\section{Some Lemmas}

We need the following lemmas.

Lemma 1 ([2]). Let $s>-1$. If $r, t>s+n+1$, then for all $a, w \in B$, we have

$$
\begin{aligned}
& \int_{B} \frac{\left(1-|z|^{2}\right)^{s} d \nu(z)}{|1-\langle z, w\rangle|^{r}|1-\langle z, a\rangle|^{t}} \\
& \quad \leq \frac{C}{\left(1-|w|^{2}\right)^{r-s-n-1}|1-\langle a, w\rangle|^{t}}+\frac{C}{\left(1-|a|^{2}\right)^{t-s-n-1}|1-\langle a, w\rangle|^{r}} .
\end{aligned}
$$

Lemma 2 ([8, Theorem 1.12]). Suppose $c$ is real and $t>-1$. Then the integrals

$$
I_{c}(z)=\int_{S} \frac{d \sigma(\zeta)}{|1-\langle z, \zeta\rangle|^{n+c}}, \quad z \in B
$$

and

$$
J_{c, t}(z)=\int_{B} \frac{\left(1-|w|^{2}\right)^{t} d \nu(w)}{|1-\langle z, w\rangle|^{n+1+t+c}}, \quad z \in B,
$$

have the following asymptotic properties:

(1) If $c<0$, then $I_{c}$ and $J_{c, t}(z)$ are both bounded in $B$;

(2) If $c=0$, then

$$
I_{c}(z) \sim J_{c, t}(z) \sim \log \frac{1}{1-|z|^{2}} \quad \text { as } \quad|z| \rightarrow 1 ;
$$

(3) If $c>0$, then

$$
I_{c}(z) \sim J_{c, t}(z) \sim\left(1-|z|^{2}\right)^{-c} \quad \text { as }|z| \rightarrow 1 .
$$

Lemma $3([1,6]) \cdot \mu$ is an $s$-Carleson measure if and only if

$$
\sup _{a \in B} \int_{B}\left(\frac{1-|a|^{2}}{|1-\langle w, a\rangle|^{2}}\right)^{n s} d \mu(w)<\infty ;
$$


and $\mu$ is a vanishing $s$-Carleson measure if and only if

$$
\lim _{|a| \rightarrow 1} \int_{B}\left(\frac{1-|a|^{2}}{|1-\langle w, a\rangle|^{2}}\right)^{n s} d \mu(w)=0 .
$$

Lemma 4 ([1, Theorem 1,2]). Suppose $n>1$ and $\frac{n-1}{n}<s \leq 1$, then $f \in Q_{s}$ if and only if $|\mathcal{R} f(z)|^{2}\left(1-|z|^{2}\right)^{n s+2} d \lambda(z)$ is an $s$-Carleson measure; $f \in Q_{s, 0}$ if and only if $|\mathcal{R} f(z)|^{2}\left(1-|z|^{2}\right)^{n s+2} d \lambda(z)$ is a vanishing $s$-Carleson measure.

Lemma 5 ([4, Proposition 5.1.2]). The triangle inequality

$$
|1-\langle a, c\rangle|^{\frac{1}{2}} \leq|1-\langle a, b\rangle|^{\frac{1}{2}}+|1-\langle b, c\rangle|^{\frac{1}{2}}
$$

holds for all $a, b, c \in \bar{B}$.

Lemma 6. If $s \geq 0$ and $t-n s \geq 0$, and $\mu$ is a vanishing $s$-Carleson measure on $B$, then

$$
\lim _{|a| \rightarrow 1} \int_{B} \frac{\left(1-|a|^{2}\right)\left(1-|z|^{2}\right)^{t-n s} d \mu(z)}{|1-\langle z, a\rangle|^{t+1}}=0 .
$$

Proof. Because a vanishing $s$-Carleson measure must be an $s$-Carleson measure, we have $\mu(B(\zeta, r)) \leq A r^{n s}, A>0$, for $\zeta \in S$ and $r>0$. Meanwhile, for $\varepsilon>0$, there exists an $r_{0} \in(0,1)$ such that $\mu(B(\zeta, r)) \leq \varepsilon r^{n s}$ for $\zeta \in S$ and $0<r<r_{0}$. For $j=1,2, \cdots, \zeta \in S$ and $r>0$, denote $E_{j, \zeta}(r)=B\left(\zeta, 2^{j+1} r\right) \backslash B\left(\zeta, 2^{j} r\right)$.

Let $a \in B, r=1-|a|<r_{0} / 4$ and $\zeta=a /|a|$. Then,

$$
|1-\langle a, \zeta\rangle|=1-|a|=r .
$$

There exists a positive integer $m$ such that $2^{m+1} r<r_{0} \leq 2^{m+2} r$. It is obvious that $m \geq 1$ and $m \rightarrow \infty$ as $|a| \rightarrow 1$. Then,

$$
\mu(B(\zeta, 2 r)) \leq \varepsilon(2 r)^{n s},
$$

$\mu\left(E_{j, \zeta}(r)\right) \leq \varepsilon\left(2^{j+1} r\right)^{n s} \quad$ if $j \leq m, \quad \mu\left(E_{j, \zeta}(r)\right) \leq A\left(2^{j+1} r\right)^{n s} \quad$ if $j>m$,

$$
\begin{gathered}
\left(1-|z|^{2}\right) \leq 2(1-|z|) \leq 2|1-\langle z, \zeta\rangle|<4 r \quad \text { if } \quad z \in B(\zeta, 2 r), \\
\left(1-|z|^{2}\right) \leq 2(1-|z|) \leq 2|1-\langle z, \zeta\rangle|<2^{j+2} r \quad \text { if } \quad z \in E_{j, \zeta}(r),
\end{gathered}
$$


and, by Lemma 5 , the definition of $E_{j, \zeta}(r)$ and (2.1),

$|1-\langle z, a\rangle|^{\frac{1}{2}} \geq|1-\langle z, \zeta\rangle|^{\frac{1}{2}}-|1-\langle a, \zeta\rangle|^{\frac{1}{2}} \geq\left(2^{\frac{j}{2}}-1\right) r^{\frac{1}{2}} \geq 2^{\frac{j-4}{2}} r^{\frac{1}{2}} \quad$ if $z \in E_{j, \zeta}(r)$.

Thus, by (2.2)- (2.6),

$$
\begin{aligned}
\int_{B} & \frac{\left(1-|a|^{2}\right)\left(1-|z|^{2}\right)^{t-n s}}{|1-\langle z, a\rangle|^{t+1}} d \mu(z) \\
& =\left(\int_{B(\zeta, 2 r)}+\sum_{j=1}^{m} \int_{E_{j, \zeta}(r)}+\sum_{j=m+1}^{\infty} \int_{E_{j, \zeta}(r)}\right) \frac{\left(1-|a|^{2}\right)\left(1-|z|^{2}\right)^{t-n s}}{|1-\langle z, a\rangle|^{t+1}} d \mu(z) \\
& \leq \frac{2 r(4 r))^{t-n s}(2 r)^{n s} \varepsilon}{r^{t+1}}+\sum_{j=1}^{m} \frac{2 r\left(2^{j+2} r\right)^{t-n s}\left(2^{j+1} r\right)^{n s} \varepsilon}{\left(2^{j-4} r\right)^{t+1}} \\
& +\sum_{j=m+1}^{\infty} \frac{2 r\left(2^{j+2} r\right)^{t-n s}\left(2^{j+1} r\right)^{n s} A}{\left(2^{j-4} r\right)^{t+1}} \\
& \leq C\left(\varepsilon+A \sum_{j=m+1}^{\infty} \frac{1}{2^{j}}\right)=C\left(\varepsilon+\frac{A}{2^{m}}\right) .
\end{aligned}
$$

Since $m \rightarrow \infty$ as $|a| \rightarrow 1$, we have

$$
\int_{B} \frac{\left(1-|a|^{2}\right)\left(1-|z|^{2}\right)^{t-n s}}{|1-\langle z, a\rangle|^{t+1}} d \mu(z)<C \varepsilon
$$

if $|a|$ is close to 1 sufficiently. This completes the proof.

Lemma 7 ([8, Theorem 2.2]). If $\alpha>-1$ and $f \in A_{\alpha}^{1}$, then

$$
f(z)=\int_{B} \frac{f(w) d \nu_{\alpha}(w)}{(1-\langle z, w\rangle)^{n+1+\alpha}}
$$

for all $z \in B$.

Lemma 8 ([8, Lemma 6.3]). Suppose $0<p<\infty, n+\alpha$ is not a negative integer, $N$ is a positive integer satisfying $N p>n$, and $f$ is holomorphic in $B$. Then $f \in B_{p}$ if and only if the function

$$
F_{N}(z)=\left(1-|z|^{2}\right)^{N} \mathcal{R}^{\alpha, N} f(z)
$$

belongs to $L^{p}(B, d \lambda)$. 


\section{Results and Proofs}

Let $A$ be a subspace of $\mathcal{B}$ and $f \in \mathcal{B}$. We denote the distance in $\mathcal{B}$ of $f$ to $A$ by $d_{\mathcal{B}}(f, A)$. For $f \in H(B)$ and $\varepsilon>0$, let $\Omega_{\varepsilon}(f)=\{z \in B$ : $\left.|\mathcal{R} f(z)|\left(1-|z|^{2}\right) \geq \varepsilon\right\}$ and let $\chi_{\Omega_{\varepsilon}(f)}$ be the characteristic function of the set $\Omega_{\varepsilon}(f)$. In these notations, our main result is formulated as follows.

Theorem 1. Let $n \geq 2, \frac{n-1}{n}<s \leq 1,0 \leq t<\infty$, and $f \in \mathcal{B}$. Then, the following quantities are equivalent:

(i) $d_{1}=d_{\mathcal{B}}\left(f, Q_{s}\right)$;

(ii) $d_{2}=\inf \left\{\varepsilon: \chi_{\Omega_{\varepsilon}(f)}(z)\left(1-|z|^{2}\right)^{n s} d \lambda(z)\right.$ is an s-Carleson measure $\}$;

(iii) $d_{3}=\inf \left\{\varepsilon: \sup _{a \in B} \int_{\Omega_{\varepsilon}(f)}|\mathcal{R} f(z)|^{t}\left(1-|z|^{2}\right)^{t}\left(1-\left|\varphi_{a}(z)\right|^{2}\right)^{n s} d \lambda(z)<\right.$ $\infty\}$;

(iv) $d_{4}=\inf \left\{\varepsilon: \sup _{a \in B} \int_{\Omega_{\varepsilon}(f)}|\mathcal{R} f(z)|^{t}\left(1-|z|^{2}\right)^{t} G(z, a)^{s} d \lambda(z)<\infty\right\}$;

(v) $d_{5}=\inf \left\{\varepsilon: \sup _{a \in B} \int_{\Omega_{\varepsilon}(f)}|\nabla f(z)|^{t}\left(1-|z|^{2}\right)^{t} G(z, a)^{s} d \lambda(z)<\infty\right\}$;

(vi) $d_{6}=\inf \left\{\varepsilon: \sup _{a \in B} \int_{\Omega_{\varepsilon}(f)}|\widetilde{\nabla} f(z)|^{t} G(z, a)^{s} d \lambda(z)<\infty\right\} ;$

(vii) $d_{7}=\inf \left\{\varepsilon: \sup _{a \in B} \int_{\Omega_{\varepsilon}(f)}|\nabla f(z)|^{t}\left(1-|z|^{2}\right)^{t}\left(1-\left|\varphi_{a}(z)\right|^{2}\right)^{n s} d \lambda(z)<\right.$ $\infty\}$

(viii ) $d_{8}=\inf \left\{\varepsilon: \sup _{a \in B} \int_{\Omega_{\varepsilon}(f)}|\widetilde{\nabla} f(z)|^{t}\left(1-\left|\varphi_{a}(z)\right|^{2}\right)^{n s} d \lambda(z)<\infty\right\}$.

Proof. First we will prove that $d_{1} \leq C d_{2}$. Let $\varepsilon$ be a positive number such that $\chi_{\Omega_{\varepsilon}(f)}(z)\left(1-|z|^{2}\right)^{n s} d \lambda(z)$ is an $s$-Carleson measure. Since $f \in \mathcal{B}$, it is easy to see that $\mathcal{R} f(z) \in A_{\alpha}^{1}$ for any $\alpha>0$. Let $\alpha>0$ be given. According to Lemma 7, we have

$$
\mathcal{R} f(z)=\int_{B} \frac{\mathcal{R} f(w) d \nu_{\alpha}(w)}{(1-\langle z, w\rangle)^{n+1+\alpha}}, \quad z \in B
$$

Since $\mathcal{R} f(0)=0$, we have

$$
\mathcal{R} f(z)=\int_{B} \mathcal{R} f(w)\left(\frac{1}{(1-\langle z, w\rangle)^{n+1+\alpha}}-1\right) d \nu_{\alpha}(w), \quad z \in B .
$$

It follows that

$$
f(z)-f(0)=\int_{0}^{1} \frac{\mathcal{R} f(t z)}{t} d t=\int_{B} \mathcal{R} f(w) L(z, w) d \nu_{\alpha}(w),
$$


where the kernel

$$
L(z, w)=\int_{0}^{1}\left(\frac{1}{(1-t\langle z, w\rangle)^{n+1+\alpha}}-1\right) \frac{d t}{t} .
$$

Let $f(z)=f_{1}(z)+f_{2}(z)$, where

$$
f_{1}(z)=f(0)+\int_{\Omega_{\varepsilon}(f)} \mathcal{R} f(w) L(z, w) d \nu_{\alpha}(w)
$$

and

$$
f_{2}(z)=\int_{B \backslash \Omega_{\varepsilon}(f)} \mathcal{R} f(w) L(z, w) d \nu_{\alpha}(w) .
$$

Since

$$
\mathcal{R} L(z, w)=\int_{0}^{1} \frac{(n+1+\alpha)\langle z, w\rangle d t}{(1-t\langle z, w\rangle)^{n+1+\alpha+1}}=\frac{1}{(1-\langle z, w\rangle)^{n+1+\alpha}}-1,
$$

we have

$$
\begin{aligned}
\left|\mathcal{R} f_{1}(z)\right| & =\left|\int_{\Omega_{\varepsilon}(f)} \mathcal{R} f(w) \mathcal{R} L(z, w) d \nu_{\alpha}(w)\right| \\
& \leq \int_{\Omega_{\varepsilon}(f)}|\mathcal{R} f(w)|\left(\frac{1}{|1-\langle z, w\rangle|^{n+1+\alpha}}+1\right) d \nu_{\alpha}(w) \\
& \leq C\left(\int_{B} \frac{\left(1-|w|^{2}\right)^{\alpha-1} d \nu(w)}{|1-\langle z, w\rangle|^{n+1+\alpha}}+1\right)
\end{aligned}
$$

and

$$
\begin{aligned}
\left|\mathcal{R} f_{2}(z)\right| & =\left|\int_{B \backslash \Omega_{\varepsilon}(f)} \mathcal{R} f(w) \mathcal{R} L(z, w) d \nu_{\alpha}(w)\right| \\
& \leq C \varepsilon\left(\int_{B} \frac{\left(1-|w|^{2}\right)^{\alpha-1} d \nu(w)}{|1-\langle z, w\rangle|^{n+1+\alpha}}+1\right) .
\end{aligned}
$$

Thus, using Lemma 2 with $c=1$ we get

$$
\left|\mathcal{R} f_{1}(z)\right| \leq \frac{C}{1-|z|^{2}}, \quad\left|\mathcal{R} f_{2}(z)\right| \leq \frac{C \varepsilon}{1-|z|^{2}},
$$

and consequently, $f_{1} \in \mathcal{B}$ and $\left\|f_{2}\right\|_{\mathcal{B}}=\left\|f_{2}\right\|_{1} \leq C \varepsilon$, since $f_{2}(0)=0$. Further we want to prove that $f_{1} \in Q_{s}$. 
We have

$$
\begin{aligned}
I\left(f_{1}, a\right)= & \int_{B}\left|\mathcal{R} f_{1}(z)\right|^{2}\left(1-|z|^{2}\right)^{2}\left(1-\left|\varphi_{a}(z)\right|^{2}\right)^{n s} d \lambda(z) \\
\leq & \left\|f_{1}\right\|_{1} \int_{B}\left|\mathcal{R} f_{1}(z)\right|\left(1-|z|^{2}\right)\left(1-\left|\varphi_{a}(z)\right|^{2}\right)^{n s} d \lambda(z) \\
\leq & \left\|f_{1}\right\|_{1} \int_{B}\left(\int_{\Omega_{\varepsilon}(f)}|\mathcal{R} f(w)|\left(\frac{1}{|1-\langle z, w\rangle|^{n+1+\alpha}}+1\right) d \nu_{\alpha}(w)\right) \\
\leq & \quad\left\|f_{1}\right\|_{1}|| f \|_{1} \int_{\Omega_{\varepsilon}(f)} d \nu_{\alpha-1}(w) \int_{B} \frac{\left(1-|z|^{2}\right)^{n s-n}\left(1-|a|^{2}\right)^{n s} d \nu(z)}{|1-\langle z, w\rangle|^{n+1+\alpha}|1-\langle z, a\rangle|^{2 n s}} \\
& \quad+\left\|f_{1}\right\|_{1}\|f\|_{1, \alpha} \int_{B} \frac{\left(1-|a|^{2}\right)^{n s}\left(1-|z|^{2}\right)^{n s-n} d \nu(z)}{|1-\langle z, a\rangle|^{2 n s}} \\
= & \left\|f_{1}\right\|_{1}\|f\|_{1} I_{1}+\left\|f_{1}\right\|_{1}|| f \|_{1, \alpha} I_{2} .
\end{aligned}
$$

Since $\alpha>0, n \geq 2$ and $(n-1) / n<s \leq 1$, we have $n s-n>-1$, $n s-1>0, n+1+\alpha>n s-n+n+1$ and $2 n s>n s-n+n+1$. Thus, by Lemma 2 and Lemma 1 ,

$$
I_{2}=\int_{B} \frac{\left(1-|a|^{2}\right)^{n s}\left(1-|z|^{2}\right)^{n s-n} d \nu(z)}{|1-\langle z, a\rangle|^{n+1+(n s-n)+n s-1}} \leq C\left(1-|a|^{2}\right),
$$

and

$$
\begin{aligned}
I_{1} \leq & C \int_{\Omega_{\varepsilon}(f)} \frac{\left(1-|a|^{2}\right)^{n s}\left(1-|w|^{2}\right)^{n s} d \lambda(w)}{|1-\langle a, w\rangle|^{2 n s}} \\
& +C\left(1-|a|^{2}\right) \int_{\Omega_{\varepsilon}(f)} \frac{\left(1-|w|^{2}\right)^{\alpha-1} d \nu(w)}{|1-\langle a, w\rangle|^{n+1+\alpha}} .
\end{aligned}
$$

Because $\chi_{\Omega_{\varepsilon}(f)}(w)\left(1-|w|^{2}\right)^{n s} d \lambda(w)$ is an $s$-Carleson measure, by Lemma 3 , we have

$$
\sup _{a \in B} \int_{\Omega_{\varepsilon}(f)}\left(\frac{1-|a|^{2}}{|1-\langle a, w\rangle|^{2}}\right)^{n s}\left(1-|w|^{2}\right)^{n s} d \lambda(w)<\infty .
$$

Using Lemma 2 we get

$$
\left(1-|a|^{2}\right) \int_{\Omega_{\varepsilon}(f)} \frac{\left(1-|w|^{2}\right)^{\alpha-1} d \nu(w)}{|1-\langle a, w\rangle|^{n+1+\alpha}} \leq C .
$$

So $\sup _{a \in B} I\left(f_{1}, a\right)<\infty$ and, by Lemma $3,\left|\mathcal{R} f_{1}(z)\right|^{2}\left(1-|z|^{2}\right)^{n s+2} d \lambda(z)$ is an $s$-Carleson measure. Consequently, by Lemma $4, f_{1} \in Q_{s}$. We have 
proved above that $\left\|f_{2}\right\|_{\mathcal{B}} \leq C \varepsilon$. Thus, $d_{\mathcal{B}}\left(f, Q_{s}\right) \leq\left\|f-f_{1}\right\|_{\mathcal{B}}=\left\|f_{2}\right\|_{\mathcal{B}} \leq$ $C \varepsilon$. This shows that $d_{1} \leq C d_{2}$.

By Lemma 3, $\chi_{\Omega_{\varepsilon}(f)}(z)\left(1-|z|^{2}\right)^{n s} d \lambda(z)$ is an $s$-Carleson measure if and only if

$\sup _{a \in B} \int_{\Omega_{\varepsilon}(f)}\left(1-\left|\varphi_{a}(z)\right|^{2}\right)^{n s} d \lambda(z)=\sup _{a \in B} \int_{\Omega_{\varepsilon}(f)} \frac{\left(1-|a|^{2}\right)^{n s}\left(1-|z|^{2}\right)^{n s}}{|1-\langle z, a\rangle|^{2 n s}} d \lambda(z)<\infty$.

Since

$$
\varepsilon \leq|\mathcal{R} f(z)|\left(1-|z|^{2}\right) \leq\|f\|_{\mathcal{B}}, \quad z \in \Omega_{\varepsilon}(f),
$$

we obtain $d_{2}=d_{3}$. It is obvious that $d_{3} \leq d_{4}$, since $\left(1-\left|\varphi_{a}(z)\right|^{2}\right)^{n} \leq$ $C G(z, a)$. It follows from $\left(1-|z|^{2}\right)|\mathcal{R} f(z)| \leq\left(1-|z|^{2}\right)|\nabla f(z)| \leq|\widetilde{\nabla} f(z)|$ that $d_{4} \leq d_{5} \leq d_{6}$.

Now, we are going to prove that $d_{6} \leq d_{1}$. For $\varepsilon>d_{1}$, there exists a function $f_{\varepsilon} \in Q_{s}$ such that $\left\|f-f_{\varepsilon}\right\|_{\mathcal{B}}<\left(d_{1}+\varepsilon\right) / 2$. Then,

$$
\begin{aligned}
\left|\widetilde{\nabla} f_{\varepsilon}(z)\right| & \geq\left|\mathcal{R} f_{\varepsilon}(z)\right|\left(1-|z|^{2}\right) \\
& \geq|\mathcal{R} f(z)|\left(1-|z|^{2}\right)-\left|\mathcal{R}\left(f-f_{\varepsilon}\right)(z)\right|\left(1-|z|^{2}\right) \\
& \geq \varepsilon-\left(d_{1}+\varepsilon\right) / 2=\left(\varepsilon-d_{1}\right) / 2, \quad z \in \Omega_{\varepsilon}(f) .
\end{aligned}
$$

Thus,

$$
\begin{array}{rl}
\sup _{a \in B} \int_{\Omega_{\varepsilon}(f)}|\widetilde{\nabla} f(z)|^{t} & G(z, a)^{s} d \lambda(z) \\
& \leq\|f\|_{3}^{t} \sup _{a \in B} \int_{\Omega_{\varepsilon}(f)} G(z, a)^{s} d \lambda(z) \\
& \leq \frac{4\|f\|_{3}^{t}}{\left(\varepsilon-d_{1}\right)^{2}} \sup _{a \in B} \int_{\Omega_{\varepsilon}(f)}\left|\widetilde{\nabla} f_{\varepsilon}(z)\right|^{2} G(z, a)^{s} d \lambda(z)<\infty .
\end{array}
$$

This shows that $d_{6} \leq \varepsilon$. Since $\varepsilon$ may be close to $d_{1}$ arbitrarily, we obtain $d_{6} \leq d_{1}$.

We have proved that $d_{1}, d_{2}, \cdots, d_{6}$ are equivalent. It follows from

$$
\left(1-|z|^{2}\right)|\mathcal{R} f(z)| \leq\left(1-|z|^{2}\right)|\nabla f(z)| \leq|\widetilde{\nabla} f(z)|
$$

and

$$
\left(1-\left|\varphi_{a}(z)\right|^{2}\right)^{n} \leq C G(z, a)
$$

that $d_{3} \leq d_{7} \leq d_{8} \leq d_{6}$. Thus, all of $d_{1}, d_{2}, \cdots, d_{8}$ are equivalent. The proof is complete.

Corollary 1. Let $n \geq 2, \frac{n-1}{n}<s \leq 1$ and $0 \leq t<\infty$. Let $f \in \mathcal{B}$. Then the following conditions are equivalent: 
(i) $f$ is in the closure of $Q_{s}$ in $\mathcal{B}$;

(ii) $\chi_{\Omega_{\varepsilon}(f)}\left(1-|z|^{2}\right)^{n s} d \lambda(z)$ is an s-Carleson measure for every $\varepsilon>0$;

(iii) $\sup _{a \in B} \int_{\Omega_{\varepsilon}(f)}|\mathcal{R} f(z)|^{t}\left(1-|z|^{2}\right)^{t}\left(1-\left|\varphi_{a}(z)\right|^{2}\right)^{n s} d \lambda(z)<\infty$ for every $\varepsilon>0$

(iv) $\sup _{a \in B} \int_{\Omega_{\varepsilon}(f)}|\mathcal{R} f(z)|^{t}\left(1-|z|^{2}\right)^{t} G(z, a)^{s} d \lambda(z)<\infty$ for every $\varepsilon>0$;

(v) $\sup _{a \in B} \int_{\Omega_{\varepsilon}(f)}|\nabla f(z)|^{t}\left(1-|z|^{2}\right)^{t} G(z, a)^{s} d \lambda(z)<\infty$ for every $\varepsilon>0$;

(vi) $\sup _{a \in B} \int_{\Omega_{\varepsilon}(f)}|\widetilde{\nabla} f(z)|^{t} G(z, a)^{s} d \lambda(z)<\infty$ for every $\varepsilon>0$;

(vii) $\sup _{a \in B} \int_{\Omega_{\varepsilon}(f)}|\nabla f(z)|^{t}\left(1-|z|^{2}\right)^{t}\left(1-\left|\varphi_{a}(z)\right|^{2}\right)^{n s} d \lambda(z)<\infty$ for every $\varepsilon>0$

(viii) $\sup _{a \in B} \int_{\Omega_{\varepsilon}(f)}|\widetilde{\nabla} f(z)|^{t}\left(1-\left|\varphi_{a}(z)\right|^{2}\right)^{n s} d \lambda(z)<\infty$ for every $\varepsilon>0$.

In the little o-spaces $\mathcal{B}_{0}$ and $Q_{s, 0}$ we have the following results.

Theorem 2. Let $n \geq 2, \frac{n-1}{n}<s \leq 1,0 \leq t<\infty$, and $f \in \mathcal{B}$. Then the following quantities are equivalent:

(i) $d_{1}^{\prime}=d_{\mathcal{B}}\left(f, \mathcal{B}_{0}\right)$;

(ii) $d_{1}=d_{\mathcal{B}}\left(f, Q_{s, 0}\right)$;

(iii) $d_{2}=\inf \left\{\varepsilon: \chi_{\Omega_{\varepsilon}(f)}\left(1-|z|^{2}\right)^{n s} d \lambda(z)\right.$ is a vanishing s-Carleson measure $\}$;

(iv) $d_{3}=\inf \left\{\varepsilon: \lim _{|a| \rightarrow 1} \int_{\Omega_{\varepsilon}(f)}|\mathcal{R} f(z)|^{t}\left(1-|z|^{2}\right)^{t}\left(1-\left|\varphi_{a}(z)\right|^{2}\right)^{n s} d \lambda(z)=\right.$ $0\}$;

(v) $d_{4}=\inf \left\{\varepsilon: \lim _{|a| \rightarrow 1} \int_{\Omega_{\varepsilon}(f)}|\mathcal{R} f(z)|^{t}\left(1-|z|^{2}\right)^{t} G(z, a)^{s} d \lambda(z)=0\right\}$;

(vi) $d_{5}=\inf \left\{\varepsilon: \lim _{|a| \rightarrow 1} \int_{\Omega_{\varepsilon}(f)}|\nabla f(z)|^{t}\left(1-|z|^{2}\right)^{t} G(z, a)^{s} d \lambda(z)=0\right\}$;

(vii) $d_{6}=\inf \left\{\varepsilon: \lim _{|a| \rightarrow 1} \int_{\Omega_{\varepsilon}(f)}|\widetilde{\nabla} f(z)|^{t} G(z, a)^{s} d \lambda(z)=0\right\}$;

(viii) $d_{7}=\inf \left\{\varepsilon: \lim _{|a| \rightarrow 1} \int_{\Omega_{\varepsilon}(f)}|\nabla f(z)|^{t}\left(1-|z|^{2}\right)^{t}\left(1-\left|\varphi_{a}(z)\right|^{2}\right)^{n s} d \lambda(z)=\right.$ $0\}$;

(ix) $d_{8}=\inf \left\{\varepsilon: \lim _{|a| \rightarrow 1} \int_{\Omega_{\varepsilon}(f)}|\widetilde{\nabla} f(z)|^{t}\left(1-\left|\varphi_{a}(z)\right|^{2}\right)^{n s} d \lambda(z)=0\right\}$.

Proof. It is known that the closure of polynomials in $\mathcal{B}$ is just $\mathcal{B}_{0}$ (cf. $[8]$ ), and $Q_{s, 0}$ contains all polynomials. So, $\mathcal{B}_{0} \subset \overline{Q_{s, 0}}$, where $\overline{Q_{s, 0}}$ is the closure of $Q_{s, 0}$ with respect to $\mathcal{B}$. On the other hand, $Q_{s, 0} \subset \mathcal{B}_{0}$, and so $\overline{Q_{s, 0}} \subset \mathcal{B}_{0}$. Thus $\overline{Q_{s, 0}}=\mathcal{B}_{0}$, which implies that $d_{1}^{\prime}$ and $d_{1}$ are equivalent.

We can show the equivalence of $d_{1}, d_{2}, \cdots, d_{8}$ in a similar way as in the proof of Theorem 1. The only point we have to explain is the proof of 
$d_{1} \leq C d_{2}$. Note that

$$
\begin{aligned}
& I\left(f_{1}, a\right) \leq C \int_{B} \frac{\left(1-|a|^{2}\right)^{n s}}{|1-\langle a, w\rangle|^{2 n s}} \chi_{\Omega_{\varepsilon}(f)}(w)\left(1-|w|^{2}\right)^{n s} d \lambda(w) \\
& \quad+C \int_{B} \frac{\left(1-|a|^{2}\right)\left(1-|w|^{2}\right)^{\alpha+n-n s}}{|1-\langle a, w\rangle|^{n+\alpha+1}} \chi_{\Omega_{\varepsilon}(f)}(w)\left(1-|w|^{2}\right)^{n s} d \lambda(w)+C\left(1-|a|^{2}\right) .
\end{aligned}
$$

Now, since $\chi_{\Omega_{\varepsilon}(f)}\left(1-|w|^{2}\right)^{n s} d \lambda(w)$ is a vanishing $s$-Carleson measure, by Lemma 3 and Lemma 6 , we see that $I\left(f_{1}, a\right) \rightarrow 0$ as $|a| \rightarrow 1$. Therefore, by Lemma 3 and Lemma $4, f_{1} \in Q_{s, 0}$. The proof is complete.

Corollary 2. Let $n \geq 2, \frac{n-1}{n}<s \leq 1$ and let $f \in \mathcal{B}$. Then $f \in \mathcal{B}_{0}$ if and only if $\chi_{\Omega_{\varepsilon}(f)}\left(1-|z|^{2}\right)^{n s} d \lambda(z)$ is a vanishing s-Carleson measure for every $\varepsilon>0$.

Finally, we will discuss the distance formulae from Bloch functions to Besov spaces on the unit ball.

Theorem 3. Let $1 \leq p<\infty$ and $f \in \mathcal{B}$. Then the following quantities are equivalent:

(i) $d_{1}=d_{\mathcal{B}}\left(f, \mathcal{B}_{0}\right)$;

(ii) $d_{2}=d_{\mathcal{B}}\left(f, B_{p}\right)$;

(iii) $d_{3}=\inf \left\{\varepsilon: \lambda\left(\Omega_{\varepsilon}(f)\right)<\infty\right\}$, where $\lambda\left(\Omega_{\varepsilon}(f)\right)=\int_{\Omega_{\varepsilon}(f)} d \nu(z) /(1-$ $\left.|z|^{2}\right)^{n+1}$.

Proof. Because $B_{p} \subset \mathcal{B}_{0}$, in a similar way to the proof of Theorem 2, it is easy to get that $d_{1}$ is equivalent to $d_{2}$.

In order to prove $d_{2} \leq C d_{3}$, let $f_{1}(z), f_{2}(z)$ be the same as in the proof of Theorem 1. What we need to do is to show $f_{1} \in B_{p}$ for $1 \leq p<\infty$. We have

$$
f_{1}(z)=f(0)+\int_{\Omega_{\varepsilon}(f)} \mathcal{R} f(w) L(z, w) d \nu_{\alpha}(w) .
$$

Then

$$
\mathcal{R}^{\alpha, n+1} f_{1}(z)=\int_{\Omega_{\varepsilon}(f)} \mathcal{R} f(w) \mathcal{R}^{\alpha, n+1} L(z, w) d \nu_{\alpha}(w),
$$

where

$$
\mathcal{R}^{\alpha, n+1} L(z, w)=\int_{0}^{1}\left(\frac{1}{(1-t\langle z, w\rangle)^{n+1+\alpha+n+1}}-1\right) \frac{d t}{t}
$$

satisfying

$$
\left|\mathcal{R}^{\alpha, n+1} L(z, w)\right| \leq \frac{C}{|1-\langle z, w\rangle|^{n+1+\alpha+n}}
$$

for all $z$ and $w$ in $B$. 
Thus we have

$$
\begin{aligned}
& \int_{B}\left(1-|z|^{2}\right)^{n+1}\left|\mathcal{R}^{\alpha, n+1} f_{1}(z)\right| d \lambda(z) \\
& \leq \int_{B} \int_{\Omega_{\varepsilon}(f)}|\mathcal{R} f(w)|\left|\mathcal{R}^{\alpha, n+1} L(z, w)\right| d \nu_{\alpha}(w) d \nu(z) \\
& \leq C \int_{\Omega_{\varepsilon}(f)} d \nu_{\alpha-1}(w) \int_{B} \frac{C}{11-\left.\langle z, w\rangle\right|^{n+1+\alpha+n}} d \nu(z) \\
& \leq C \int_{\Omega_{\varepsilon}(f)} \frac{d \nu_{\alpha-1}(w)}{\left(1-|w|^{2}\right)^{n+\alpha}} \\
& =C \int_{\Omega_{\varepsilon}(f)} \frac{d \nu(w)}{\left(1-|w|^{2}\right)^{n+1}} .
\end{aligned}
$$

Using Lemma 8 with $p=1$ and $N=n+1$ we get $f_{1} \in B_{1} \subset B_{p}$ if $\lambda\left(\Omega_{\varepsilon}(f)\right)<\infty$.

The last matter is to prove that $d_{3} \leq d_{2}$. Since $B_{p_{1}} \subset B_{p_{2}}$, where $1 \leq p_{1}<p_{2}<\infty$, we may assume $n<p<\infty$. For $\varepsilon>d_{2}$, there exists a function $f_{\varepsilon} \in B_{p}$ such that $\left\|f-f_{\varepsilon}\right\|_{\mathcal{B}}<\left(d_{2}+\varepsilon\right) / 2$. Then

$$
\begin{aligned}
\left|\mathcal{R} f_{\varepsilon}(z)\right|\left(1-|z|^{2}\right) & \geq|\mathcal{R} f(z)|\left(1-|z|^{2}\right)-\left|\mathcal{R}\left(f-f_{\varepsilon}\right)(z)\right|\left(1-|z|^{2}\right) \\
& \geq \varepsilon-\left(d_{2}+\varepsilon\right) / 2=\left(\varepsilon-d_{2}\right) / 2, \quad z \in \Omega_{\varepsilon}(f) .
\end{aligned}
$$

By $[8$, Exercise 6.8$]$ and $f_{\varepsilon} \in B_{p}$,

$$
\int_{B}\left(1-|z|^{2}\right)^{p}\left|\mathcal{R} f_{\varepsilon}(z)\right|^{p} d \lambda(z)<\infty, \quad n<p<\infty .
$$

Thus

$$
\begin{aligned}
\lambda\left(\Omega_{\varepsilon}(f)\right) & =\int_{\Omega_{\varepsilon}(f)} \frac{d \nu(z)}{\left(1-|z|^{2}\right)^{n+1}} \\
& \leq \frac{2^{p}}{\left(\varepsilon-d_{2}\right)^{p}} \int_{\Omega_{\varepsilon}(f)}\left(1-|z|^{2}\right)^{p}\left|\mathcal{R} f_{\varepsilon}(z)\right|^{p} d \lambda(z)<\infty,
\end{aligned}
$$

which shows that $d_{3} \leq \varepsilon$. Since $\varepsilon$ may be close to $d_{2}$ arbitrarily, we have $d_{3} \leq d_{2}$. The proof is complete.

Corollary 3. Let $f \in \mathcal{B}$. Then $f \in \mathcal{B}_{0}$ if and only if $\lambda\left(\Omega_{\varepsilon}(f)\right)<\infty$ for every $\varepsilon>0$.

Acknowledgement. It is a pleasure to thank Professor R. Aulaskari, Professor H. Chen and R. Zhao for their helpful comments and suggestions. 


\section{References}

[1] Z. Hu, $Q_{p}$ spaces in the unit ball of $\mathbb{C}^{n}$ with $\frac{n-1}{n}<p \leq 1$, J. Huzhou Teachers College, 25 (2004), 1-10.

[2] J. M. Ortega and J. Fàbrega, Pointwise multipliers and corona type decomposition in BMOA, Ann. Inst. Fourier (Grenoble), 46 (1996), 111-137.

[3] C. Ouyang, W. Yang and R. Zhao, Möbius invariant $Q_{p}$ spaces associated with the Green's function on the unit ball of $\mathbb{C}^{n}$, Pacific J. Math., 182 (1998), 69-99.

[4] W. Rudin, Function Theory in the Unit Ball of $\mathbb{C}^{n}$, Springer, New York, 1980.

[5] O. Ullrich, Radial limits of M-subharmonic functions, Trans. Amer. Math. Soc., 292 (1985), 501-518.

[6] W. Yang, Carleson type measure characterizations of $Q_{p}$ spaces, Analysis, 18 (1998), 345-349.

[7] R. Zhao, Distances from Bloch functions to some Möbius invariant spaces, Ann. Acad. Sci. Fenn. Math., 33 (2008), 303-313.

[8] K. Zhu, Spaces of Holomorphic Functions in the Unit Ball, SpringerVerlag, New York, 2004.

Department of Physics and Mathematics

University of Joensuu

P. O. Box 111

FI-80101 Joensuu

Finland

(E-mail : wxu@cc.joensuu.fi)

(Received : December 2007) 


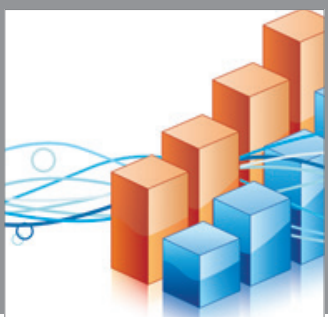

Advances in

Operations Research

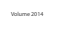

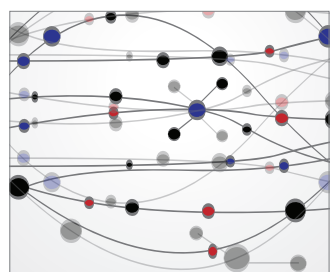

\section{The Scientific} World Journal
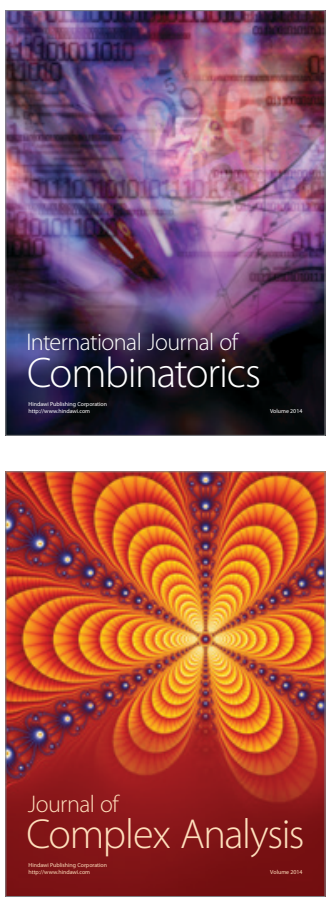

International Journal of

Mathematics and

Mathematical

Sciences
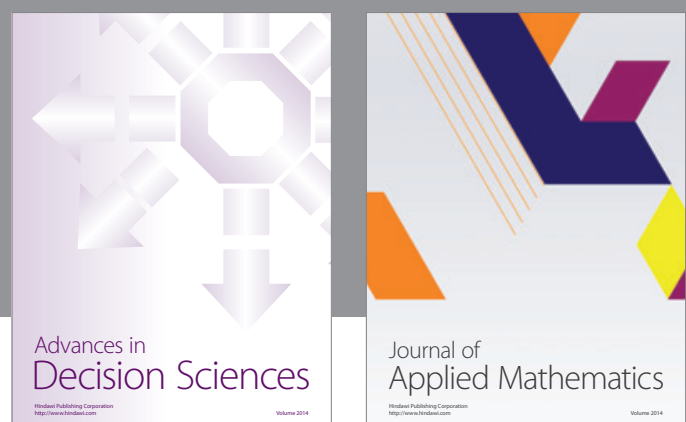

Journal of

Applied Mathematics
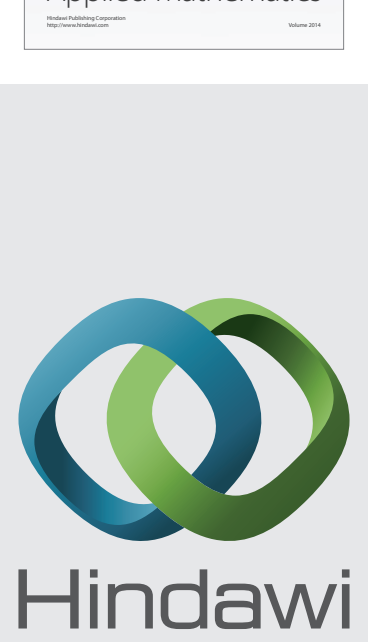

Submit your manuscripts at http://www.hindawi.com
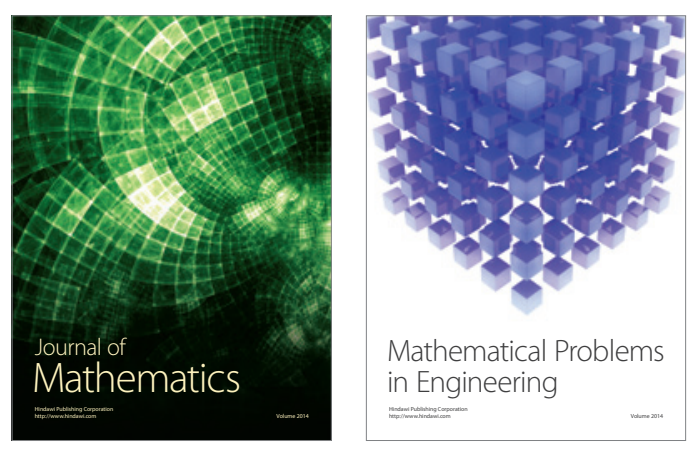

Mathematical Problems in Engineering
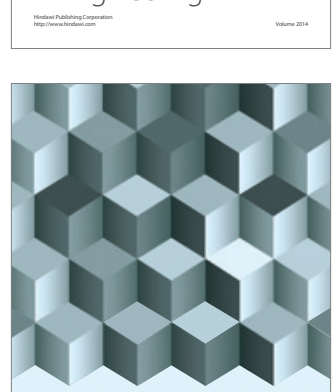

Journal of

Function Spaces
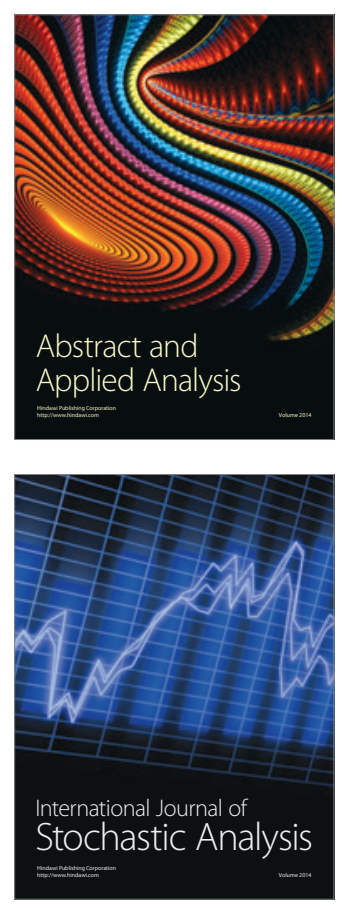

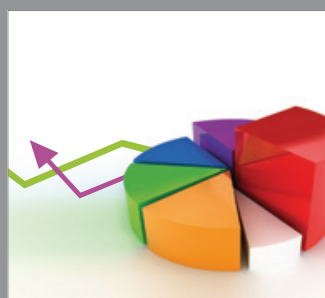

ournal of

Probability and Statistics

Promensencen
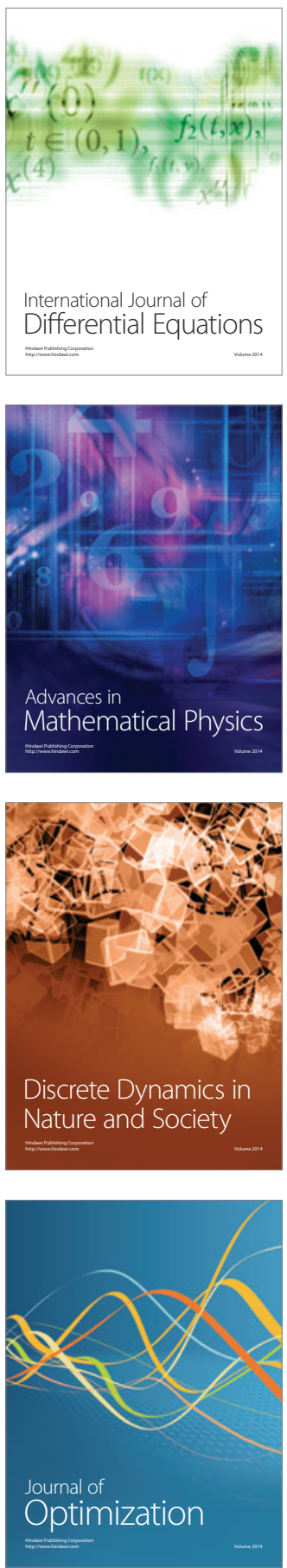